\title{
The epigenetic landscape of addiction
}

\author{
Ian Maze ${ }^{\mathrm{a}}$ and Eric J. Nestler ${ }^{\mathrm{a}}$ \\ aFishberg Department of Neuroscience, Mount Sinai School of Medicine, New York, New York \\ 10029-6574
}

\begin{abstract}
Drug-induced alterations in gene expression throughout the reward circuitry of the brain are likely components of the persistence of the drug-addicted state. Recent studies examining the molecular mechanisms controlling drug-induced transcriptional, behavioral and synaptic plasticity have indicated a direct role for chromatin remodeling in the regulation and stability of drug-mediated neuronal gene programs, and the subsequent promulgation of addictive behaviors. In this review, we discuss recent advances in our understanding of chromatin phenomena-that is, epigenetics, by one definition - that contribute to drug addiction, with the hope that such mechanistic insights may aid in the development of novel therapeutics for future treatments of addiction.
\end{abstract}

\section{Keywords}

histone; chromatin; plasticity; histone deacetylase (HDAC); histone methyltransferase (HMT); drug addiction

\section{Introduction}

Drug addiction is a debilitating chronic relapsing disorder that is characterized by pathological drug taking- and seeking- behaviors despite adverse consequences to both the drug abuser and those directly affected by their behavior ${ }^{1-3}$. Although family history represents one of the greatest risk factors for drug addiction, as well as other prevalent psychiatric disorders, the genetic basis for such illnesses remains poorly understood. With prominent advances in whole genome sequencing, the search for genetic variations underlying drug addiction is continuing at an escalating pace; however, genetic factors likely explain $\sim 50 \%$ of the risk for addiction. Therefore, much investigation has focused on the environmental components of drug addiction, which have often been much more difficult to decipher mechanistically. The central hypothesis in the field is that exposure to a wide variety of environmental stimuli, interacting with an individual's genetic constitution, determines initial responses to drugs of abuse as well as maladaptations to repeated drug exposure that underlie the transition to an addicted state. Because the behavioral abnormalities that characterize addiction are so long-lived, the field has focused on druginduced alterations in gene expression patterns throughout specific brain "reward" related regions, such as the nucleus accumbens (NAc), in assessing this potantial key mechanism for mediating addiction.

Correspondence should be addressed to Eric J. Nestler, Fishberg Department of Neuroscience, Mount Sinai School of Medicine, One Gustave L. Levy Place, Box 1065, New York, NY 10029-6574. Phone: 212-659-5656. Fax: 212-659-8510. eric.nestler@mssm.edu. 


\section{“Epigenetics:" a definition}

Interactions between genes and the environment that result in specific biological phenotypes are one way in which the term "epigenetic" 4 is used. In 1957, the idea of an "epigenetic landscape" was first described by Conrad Waddington, a developmental biologist who was interested in explaining how identical genotypes could result in such a wide variety of phenotypic variation throughout the process of development ${ }^{5}$. Later, this concept would evolve to include the additional suggestion that "potentially heritable changes in gene expression [occur] that do not involve changes in DNA sequence" ${ }^{6-8}$.

This process is exemplified throughout embryonic cellular differentiation, during which time-unique chemical signals promote the differentiation of totipotent stem cells into genotypically identical cell types displaying varied phenotypic functions ${ }^{9}$. Such events are essential for the generation of distinct cells throughout the body that express vastly different sets of genes (e.g., cardiac myocytes versus neurons). These events may help to explain how the relatively fixed human genome of $\sim 25,000$ genes ultimately shapes our experiences into the complex behavioral patterns and thoughts that make human beings distinct from other life forms ${ }^{10}$.

While many questions remain, mechanistic insight into Waddington's landscape has taken a clear molecular form with documented evidence indicating that environmental signals can be transduced to promote stable alterations in chromatin structure. These changes occur via a number of multisubunit complexes that act to regulate DNA's access to the transcriptional machinery, thus activating or repressing specific gene programs ${ }^{11}$. Such events ultimately result in the promulgation of specific gene expression patterns in response to distinct environmental cues through a combination of chromatin remodeling activity, enzymatic modification of DNA and histones, and nucleosomal histone subunit exchange ${ }^{11-13}$. The regulatory control exerted by mechanisms of chromatin remodeling, as well as the potential stability of such modifications, make epigenetic regulation a prime candidate for mediating some of the persistent alterations in transcriptional and neural plasticity that are thought to underlie aspects of drug addiction.

\section{Epigenetic mechanisms}

At its most basic level, chromatin functions to ensure the proper organization, storage and readout of genetic information with remarkable spatial and temporal precision during processes of cellular differentiation and organismal development ${ }^{10}$. The nucleosome core particle exists as the fundamental repeating unit of chromatin, each composed of $147 \mathrm{bp}$ of DNA organized in approximately two superhelical turns around an octameric core of histone proteins (two copies each of $\mathrm{H} 2 \mathrm{~A}, \mathrm{H} 2 \mathrm{~B}, \mathrm{H} 3$ and H4, or variants of these proteins) (Figure $1 \mathrm{~A})^{14}$. Structural variations of the nucleosome particle can be introduced via a number of tightly regulated mechanisms, including post-translational covalent modifications of histones, histone variant deposition, and chromatin remodeling complexes that promote altered levels of chromatin compaction, resulting in more "open" (euchromatic) versus "closed" (heterochromatic) transcriptional configurations. Such configurations typically reflect more "active" versus "inactive" states of gene expression, respectively ${ }^{11-13}$. For example, histone modifications that weaken or disrupt histone:DNA contacts, such as histone acetylation, correlate with transcriptionally active states, whereas modifications that increase histone:DNA contacts, such as histone methylation at certain basic amino acid residues, promote transcriptional repression ${ }^{13}$. Combinations of numerous post-translational modifications occurring on $\mathrm{N}$-terminal histone tails, including phosphorylation, methylation, sumoylation, and others, have been demonstrated to affect condensation of chromatin and to 
result in altered levels of gene expression in cells (Figure 1B) ${ }^{15}$. Likewise, direct methylation of cytosine bases within DNA controls the activity of the affected genes.

These events occur through a very dynamic and complex system of enzymatic events that employ various "writer/eraser" proteins to catalyze the addition or removal, respectively, of histone marks at specific substrates. "Effector" proteins with distinct "reader" modules recognize specific histone modifications, or combinations thereof, to promote various downstream events ${ }^{13,16-18}$. Ultimately, many different modifications occur at distinct histone residues throughout the genome, and these modifications are summated to determine the transcriptional output of genes into messenger RNAs and proteins as well as numerous non-coding RNAs ${ }^{15}$. One important issue to consider is the apparent stability of certain chromatin modifications. This stability is evident in X-inactivation or genetic imprinting, whereby DNA methylation promotes lifelong gene silencing ${ }^{19}$. Despite such high levels of apparent stability of some epigenetic modifications in vivo, most, if not all, forms of chromatin modifications are potentially reversible, and specific enzymes or mechanisms exist to mediate the addition or removal of associated marks ${ }^{20}$. The in vivo mechanisms that control the transiency versus stability of specific chromatin modifications remain largely unknown.

\section{Histone acetylation}

Acetylation at basic amino acid residues on histone proteins acts to reduce histone:DNA contacts through neutralization of the electrostatic interaction between negatively charged DNA and positively charged $\square$-amino groups on lysines located on N-terminal histone tails ${ }^{20}$. This modification results in a more relaxed chromatin structure to allow greater access of the transcriptional machinery to DNA. Histone acetylation is tightly regulated through the actions of numerous histone acetyltransferases (HATs) and histone deacetylases (HDACs), which respectively catalyze the addition or removal of acetyl groups on histone proteins. Genome-wide analyses have indicated a positive correlation between levels of histone acetylation at gene promoters and transcriptional activity ${ }^{21}$. Although most genomic studies have focused on acetylation on histones $\mathrm{H} 3$ and $\mathrm{H} 4$, all other histone proteins can be acetylated both on their $\mathrm{N}$-terminal tails, as well as within their globular domains ${ }^{20}$.

\section{Histone phosphorylation}

Histone phosphorylation is another post-translational modification of histones that is generally correlated with transcriptional activation. The most heavily characterized histone phosphorylation site is Serine 10 on $\mathrm{H} 3$ (H3S10). This phosphorylation event promotes association with, and stabilization of, the histone acetyltransferase (HAT), GCN5, and subsequently antagonizes repressive methylation of lysine 9 on H3 (H3K9) and the recruitment of associated co-repressors, such as heterochromatin protein $1(\mathrm{HP} 1)^{20}$. This 'blockade' of repressive $\mathrm{H} 3 \mathrm{~K} 9$ methylation is due to the fact that $\mathrm{H} 3 \mathrm{~S} 10$ phosphorylation leads to the recruitment of associated HATs, which promote acetylation at the neighboring $\mathrm{H} 3 \mathrm{~K} 9$ residue, a process referred to as phospho-acetylation. Interestingly, histone phosphorylation is often observed on the promoters of rapidly inducible, "immediate early" genes (IEGs), such as $c$-fos, following induction by either glutamate or cAMP treatment in cultured striatal neurons. Stimulation does not always result in increased acetylation, providing further evidence that, although these activating marks are associated, they can function independently to drive downstream transcriptional events ${ }^{22,23}$.

\section{Histone methylation}

Unlike acetylation, histone methylation is unique in terms of its relative stability and complexity of regulation ${ }^{24}$. As with the addition of acetyl groups to $\mathrm{N}$-terminal histone tails, methyl groups can be added to the $\varepsilon$-amino groups of lysine and arginine residues through 
the high-energy enzymatic donor S-adenosyl methionine (SAM). Methylation does not alter the charge of target amino acids and can exist in a multivalent state, however (i.e., histone lysine and arginine residues can be mono-, di- or tri-methylated $)^{24}$. Furthermore, both the specific amino acid residues being methylated, as well as the valence of methylation at those residues, are controlled by distinct histone methyltransferases (HMT) and histone demethylases (HDMs). These enzymatic activities ultimately determine whether a particular methylation event is transcriptionally activating or repressive at a given gene promoter. For example, trimethylation of $\mathrm{H} 3$ lysines 4 (H3K4me3) and 36 (H3K36me3) are highly associated with transcriptional initiation and elongation, respectively, and often correlate with increased levels of transcriptional activity. On the other hand, di- and tri-methylation on $\mathrm{H} 3$ lysines 9 (H3K9me2/3) and 27 (H3K27me2/3) are generally associated with transcriptional repression and are distributed throughout both euchromatic and heterochromatic regions of the genome ${ }^{24}$. Transcriptional silencing by $\mathrm{H} 3 \mathrm{~K} 9 \mathrm{me} 3$ is regulated, in part, by recruitment of co-repressive proteins, such as HP1, which may act to directly influence chromatin structural dynamics, resulting in increased chromatin compaction $^{24}$.

It should be noted that such generalized roles for these modifications are likely to represent oversimplification of their functions. For example, increased levels of H3K4 methylation (H3K4me) in the proximal promoter of a gene do not necessarily result in increased levels of expression of that gene. $\mathrm{H} 3 \mathrm{~K} 4 \mathrm{me}$ has also been demonstrated to act as a transcriptional pause signal, tethering HDAC complexes to influence a gene's level of transcriptional priming for future activation ${ }^{25}$. Furthermore, the existence of "bivalent domains," characterized by a configuration of both activating (H3K4me) and repressive (H3k27me) marks at gene promoters, as well as the fact that selected combinations of numerous posttranslational modifications of $\mathrm{H} 3 \mathrm{~N}$-terminal tails summate to influence gene expression profiles (e.g., increased levels of $\mathrm{H} 3$ and $\mathrm{H} 4$ acetylation, as well as high levels of $\mathrm{H} 3 \mathrm{~K} 4 \mathrm{me} 3$, at gene promoter regions often result in active transcription), suggest a high degree of complexity in the final readout of histone methylation marks ${ }^{26}$. Thus, histone methylation ultimately provides cells with precise control over the transcriptional activity of individual genes through intricate combinatorial possibilities that are still under active exploration.

\section{DNA methylation}

DNA methylation represents a unique epigenetic modification, in that it involves enzymatic addition of methyl groups to DNA cytosine bases, as opposed to modifications of histone proteins. DNA methylation is required for proper organismal development, genetic imprinting, X-chromosome inactivation, and tissue-specific gene expression, as well as other activities $^{27}$. DNA methylation is believed to be a repressive modification. Methylation at specific cytosine-guanine dinucleotides ( $\mathrm{CpG}$ islands) has been shown to interfere with transcription factor binding to target sequences through the recruitment of numerous corepressor complexes. Such repressive complexes are recruited to methylated DNA through the actions of methyl-binding domain-containing proteins, such as MeCP2 and MBD1, which function to further stabilize additional co-repressors (e.g., HDACs) at gene promoters. Binding of the transcription factor CREB, for example, can be affected by DNA methylation at gene promoters, since the consensus cAMP response element (CRE) sequence contains a $\mathrm{CpG}$ island, which, when methylated, prevents CREB binding to its target sequences ${ }^{28}$. Further evidence to support the importance of DNA methylation in normal brain development comes from studies demonstrating that mutations in MeCP2 result in an autism spectrum disorder, Rett syndrome. It should be noted, however, that recent studies have indicated that $\mathrm{MeCP} 2$ can act as both a repressor and activator of gene transcription. Its actions can be found throughout the genome, suggesting that the context and localization of 
DNA methylation are important contributing factors to its final effects on gene transcription $^{29-31}$.

\section{Epigenetics and drug addiction}

Repeated exposure to numerous drugs of abuse alters gene expression profiles throughout the reward circuitry of the brain. Recent data suggest that repeated exposure to the psychostimulant cocaine promotes alterations in histone acetylation, phosphorylation and methylation levels, as well as DNA methylation levels, in the NAc. It is thus hypothesized that these modifications may be involved in mediating drug-induced behaviors ${ }^{32,33}$.

Acute cocaine administration, for example, was shown to rapidly (i.e., effects appear within 30 minutes of drug treatment) and transiently (i.e., effects disappear by 3 hours following drug exposure) increase $\mathrm{H} 4$ acetylation at the proximal promoters of the IEGs $c$-fos and fosb in the NAc, this timecourse is thus consistent with their kinetics of induction following cocaine administration ${ }^{34}$. Interestingly, although several control gene promoters were unaffected by acute cocaine treatment (e.g., $\beta$-tubulin and histone H4), global H4 acetylation and $\mathrm{H} 3$ phospho-acetylation were transiently increased by drug treatment, suggesting that global alterations in acetylation may act to influence the transcriptional profiles of a subset of genes involved in mediating cocaine-induced behaviors ${ }^{34,35}$. Such global changes in histone marks have also been observed in the prefrontal cortex (PFC) of rats in response to adolescent cocaine exposure, in which both the activating mark, H3K4me3, and the repressive mark, H3K27me3, were found in reduced intensity, perhaps suggesting that that these global decreases are occurring on distinct promoters to drive altered behavioral responses to the drug. However, evidence for such a conclusion is still lacking ${ }^{36}$.

Unlike acute drug exposure, chronic cocaine exposure, either experimenter-delivered or selfadministered, can persistently induce expression of a distinct set of genes in the NAc (e.g., $b d n f$ and $c d k 5)^{37,38}$. In line with such stable changes in gene expression, levels of histone $\mathrm{H} 3$ acetylation increased at the promoters of both $c d k 5$ and $b d n f$ for 1 to 7 days following drug exposure ${ }^{34}$. Stable alterations in acetylation and gene expression have also been demonstrated to occur in the PFC following relatively long periods of withdrawal from cocaine self-administration ${ }^{39}$. Although under studied, the persistence of gene expression changes throughout multiple limbic forebrain regions following chronic exposure to drugs of abuse suggests that chromatin modifications may play roles in regulating transcriptional plasticity in these other brain structures as well.

Following initial studies aimed at analyzing the effects of drug exposure on chromatin regulation at specific gene promoters via quantitative chromatin immunoprecipitation (ChIP) assays, it was important to utilize genome-wide techniques to characterize such druginduced histone modifications across every gene promoter throughout the genome. To do so, chromatin that was selectively immunoprecipitated using antibodies directed against polyacetylated $\mathrm{H} 3$ or $\mathrm{H} 4$, or methylated $\mathrm{H} 3$ ( $\mathrm{H} 3 \mathrm{~K} 9 \mathrm{me} 2$ and $\mathrm{H} 3 \mathrm{~K} 27 \mathrm{me} 2$ ), was hybridized to genome-wide promoter microarrays (ChIP-chip). These experiments allowed us to compare enrichment of these marks at promoters across the genome from the NAc of cocaine naïve versus cocaine experienced animals ${ }^{40}$. Genomic binding patterns of the drug-induced transcription factors CREB and $\triangle \mathrm{FosB}$ were also assessed and compared to drug-induced chromatin modifications in order to better identify putative target genes that may mediate persistent behavioral syndromes following chronic drug exposure. These high-resolution genomic maps of histone modifications and transcription factor binding patterns provide new insight into the function of such events in in vivo regulation of neuronal-specific transcriptional responses to drugs of abuse. 
For example, these studies directly addressed earlier findings indicating that acute cocaine treatment results in increased $\mathrm{H} 4$ acetylation at acutely regulated gene promoters, whereas $\mathrm{H} 3$ acetylation appears to predominate at chronically induced promoters ${ }^{34}$. ChIP-chip analyses revealed that many more genes display hyperacetylation on $\mathrm{H} 3$ in comparison to $\mathrm{H} 4$ (Figures 2A and 2B), consistent with previous reports. Numerous previouslyunidentified promoters were also shown to be hyperacetylated at $\mathrm{H} 4$ following chronic cocaine treatment, indicating that both of these marks may be involved in repeated cocaineinduced gene transcription ${ }^{40}$. Interestingly, only a very small subset of gene promoters displayed hyperacetylation at both $\mathrm{H} 3$ and $\mathrm{H} 4$ following chronic cocaine administration, suggesting that, although both modifications may be important for cocaine's effects on gene expression, these two marks most likely function independently on distinct subsets of genes to promote differential downstream effects. Another study of a limited number of genes using seizure models found that most chronically induced transcripts displayed $\mathrm{H} 3$, and not $\mathrm{H} 4$, hyperacetylation. A subset of these genes, consisting mainly of IEGs, were also induced via acute stimulation and frequently displayed increased levels of $\mathrm{H} 4$ acetylation at their promoters ${ }^{41}$. These data indicate that the apparent $\mathrm{H} 4$ to $\mathrm{H} 3$ acetylation switch following acute versus chronic cocaine or seizures might occur only at the promoters of specific types of genes, such as IEGs. Another interesting discovery from these data was the finding that a very limited number of genes displayed hypoacetylation on either $\mathrm{H} 3$ or $\mathrm{H} 4$ following chronic cocaine. These data suggest that, in general, cocaine acts to enhance patterns of gene expression in the NAc through active acetylation, or inhibition of deacetylation, to promote transcriptional plasticity and expression of numerous drug-related transcripts, some of which may be important in the development of cocaine-induced behaviors ${ }^{40}$.

Furthermore, analyses of $\triangle \mathrm{FosB}$ and phospho-CREB binding indicate an overall enhancement of transcription factor recruitment to gene promoters following cocaine exposure with very little overlap between levels of transcription factor binding and $\mathrm{H} 3 \mathrm{~K}$ / K27me2 levels at active gene promoters (Figure 2C) ${ }^{40}$. Interestingly, although H3K9/ $\mathrm{K} 27 \mathrm{me} 2$ levels varied across gene promoters after cocaine treatment, there was very little overlap between cocaine-induced transcripts displaying both increased $\mathrm{H} 3 / \mathrm{H} 4$ acetylation and H3K9/K27me2 (Figures 2A and 2B). These two marks, which differ significantly in terms of their respective stability, may mediate transcriptional events at distinct subsets of genes ${ }^{40}$. Taken together, these types of analyses, along with next-generation sequencing technologies, will allow for a much better understanding of the genomic chromatin signature that results from chronic treatment with drugs of abuse. Such analyses will aid in the identification of gene targets that may be important in regulating various aspects of the addictive behaviors.

Some of the first evidence to suggest a role for epigenetic phenomena in the development of addictive-like behaviors came from studies demonstrating that pharmacological HDAC inhibition, or genetic manipulation of specific repressive class IIa HDACs, dramatically affected animals' behavioral responses to cocaine ${ }^{34}$. Systemic administration of two nonspecific HDAC inhibitors (HDACis), sodium butyrate (NaBut) or trichostatin A (TSA), as well as intra-NAc delivery of suberoylanilide hydroxamic acid (SAHA), a more specific HDACi, significantly potentiated cocaine's behavioral effects as measured by the conditioned place preference (CPP) measure of drug reward (Figure $3 \mathrm{~A})^{34,42}$. Similar effects of HDACis on locomotor sensitization can be observed with dopamine D1-specific agonists, amphetamine, morphine and ethanol ${ }^{43-45}$. Interestingly, recent studies suggest that HDAC inhibition potentiates extinction from CPP, and reduces reinstatement to this behavior. HDAC inhibition, and thus increased acetylation, may thus generally function to enhance behavioral saliency and promote adaptive responses to a large variety of environmental stimuli $^{46,47}$. Consistent with these findings, mice that are genetically depleted of the HAT, 
CBP, were shown to exhibit reduced behavioral sensitivity to cocaine and to display reduced acetylation at the $f o s b$ promoter ${ }^{48}$.

Reducing acetylation through viral-mediated transfer and overexpression of specific HDACs in the NAc (i.e., HDAC4 and 5, but not HDAC9) decreased CPP in a way that depended on catalytically active enzyme ${ }^{42}$. On the other hand, global knockout of HDAC5 in animals enhanced the rewarding properties of cocaine (Figure 3B), an effect that can be rescued by targeted overexpression of this enzyme in the NAc. The finding that certain HDACs, including HDAC5, may specifically control aspects of drug-induced behavior prompted further investigations into the mechanisms through which cocaine may exert its effects on histone acetylation following drug exposure ${ }^{42}$. It was later observed that chronic, but not acute, exposure to cocaine induced HDAC5 phosphorylation and nuclear export of the enzyme via the chaperone protein 14-3-3. This event requires the enzymatic activity of CaMKIIa, which has also been demonstrated to be epigenetically and transcriptionally induced by psychostimulant exposure ${ }^{42}$. These events ultimately result in hyperacetylation and increased expression of HDAC5 target genes in the NAc, phenomena which have also been observed in animals' chronically self-administering cocaine ${ }^{49}$. Based on previous transcriptional data, such increased histone acetylation in the NAc presumably contributes to sensitized behavioral responses observed following drug treatment.

It should be noted that, although these biochemical changes occur both in animals receiving experimenter-delivered as well as self-administered cocaine, systemic HDAC inhibition promotes differential behavioral responses to cocaine in self-administration paradigms. These effects depend critically on the time at which HDACi treatment is initiated. For example, rats that are treated with the HDACi, TSA, before each fixed ratio 1 (FR1) scheduled self-administration session display significantly reduced numbers of cocaine selfinjections during subsequent training sessions, indicating that these animals find cocaine to be less effective in this reinforcement paradigm ${ }^{50}$. Interestingly, however, animals that are trained to self-administer cocaine prior to HDAC inhibition with the non-specific HDACi, $\mathrm{NaBut}$ (i.e., NaBut is delivered just prior to the test session while animals are in the maintenance phase of self-administration behavior), display increased lever pressing for cocaine, suggesting that these animals find cocaine to be more reinforcing. This result is consistent with data indicating enhanced behavioral responses to drugs of abuse following HDACi treatment ${ }^{51}$. Similar results were also observed in rats receiving daily intra-NAc infusions of SAHA. HDAC inhibition caused an upward shift in the dose response curve under a fixed-ratio schedule and increased the break point under a progressive-ratio schedule, indicating enhanced motivation for self-administered drug 52 .

Although difficult to explain, the time-course of HDAC inhibition, and the resultant effects on cocaine-induced behaviors, mimic findings which demonstrate that knockout of HDAC5 alone in animals is not sufficient to promote increased reward-like behaviors. Nevertheless, HDAC5 knockout animals that have received prior cocaine exposure display dramatically enhanced CPP (Figure 3B), indicating that HDAC5 inhibition, and thus increased histone acetylation, provides a potential molecular component of the "switch" that governs the transition to the chronically `addicted' state ${ }^{42}$. Interestingly, unpublished data indicate a direct repressive role for HDAC5 in the regulation of dendritic spine plasticity in the NAc. These data further support roles for activating patterns of chromatin regulation in the enhancement of synaptic plasticity. In accordance with these findings, increased acetylation has been observed at numerous gene promoters of proteins known to play a role in synaptic activity following chronic psychostimulant administration, including NFkB subunits, Cdk5 and CaMKIIa ${ }^{34,52,53}$. 
Recently, the class III HDACs, sirtuin 1 and sirtuin 2 (SIRT1 and SIRT2), were identified by genome wide ChIP-chip analyses as potential targets of increased $\mathrm{H} 3$ acetylation following chronic cocaine administration. Both genes were transcriptionally induced by the drug, leading to their increased enzymatic activity in the $\mathrm{NAc}^{40}$. Further examination of these genes' promoters revealed a possible role for the transcription factor $\Delta \mathrm{FosB}$ in the regulation of sirtuin expression and a potential function for these enzymes in the development of cocaine-induced behaviors. Pharmacological manipulation of sirtuin activity through administration of the sirtuin activator, resveratrol, or the sirtuin inhibitor, sirtinol, provided opposing patterns of cocaine-induced behaviors. Sirtuin inhibition decreased conditioned place preference for the drug and decreased cocaine-self administration, Sirtuin activation, on the other hand, enhanced rewarding responses to the drug perhaps by increasing the electrical excitability of NAc neurons ${ }^{40}$. Since sirtuins are known to act both directly and indirectly to drive increased transcriptional repression in cells, these findings might seem to contradict previous data indicating that global increases in histone acetylation following cocaine exposure result in enhanced transcriptional plasticity, and therefore heightened levels of drug-induced behavioral responsivity. Interestingly, sirtuins, like other chromatin remodeling enzymes, have been demonstrated to act on numerous non-histone substrates, some of which may be involved in the development of cocaine-induced behaviors ${ }^{40,54}$. For example, ex vivo application of sirtinol to NAc slices prevents the induction of phospho-ERK (pERK) signaling in response to cellular depolarization ${ }^{40}$. Based on literature demonstrating roles for $\mathrm{pERK}$ signaling in response to repeated cocaine exposure, one would predict that inhibition of such signaling would result in decreased behavioral responses to the drug ${ }^{55}$. Indeed, animals receiving intra-NAc delivery of sirtinol display reduced conditioned place preference for cocaine.

Histone $\mathrm{H} 3$ phosphorylation and phospho-acetylation, which are considered to be activating marks of transcription, have also recently been shown to be important in the development of cocaine-induced behaviors. For example, cocaine administration rapidly induces $\mathrm{H3}$ phosphorylation and phospho-acetylation in the striatum ${ }^{34,35}$. The H3 kinase MSK1, a known downstream member MAP kinase signaling, was demonstrated to mediate this cocaine-induced increase in $\mathrm{H} 3$ phosphorylation ${ }^{35}$. Furthermore, depletion of MSK1 results in reduced locomotor responses to the drug, consistent with a role for histone phosphorylation events in mediating drug-induced behavioral sensitivity. The protein phosphatase inhibitor, DARPP32, has also been shown to regulate changes in histone phosphorylation in striatum in response to cocaine administration ${ }^{56}$.

Corresponding data suggest that loss of MSK1 blocks the acute induction of the immediate early gene (IEG) $c$-fos, which has long been demonstrated to be transcriptionally induced in response to acute cocaine exposure $34,35,57$. This acute induction of $c$-fos can be potentiated by HDACis. However, chronic psychostimulant administration has been shown to desensitize $c$-fos expression, an event that results, in part, from recruitment of a corepressive $\triangle$ FosB:HDAC1 (class I HDAC) complex to the $c$-fos promoter ${ }^{35,58}$. Since many acute behavioral responses to cocaine can require appropriate $c$-fos activation, it follows that loss of MSK1, which reduces cocaine-induced $\mathrm{H} 3$ phosphorylation in striatum, as well as subsequent cocaine-mediated locomotor sensitivity, results in the loss of cocaine-mediated $c$-fos transcriptional activity. $c$-fos is acutely induced by a single cocaine exposure and is desensitized following repeated psychostimulant administration. Both events may promote drug-induced behaviors. It is thus likely that such differential expression patterns result from different modes of chromatin regulation at its promoter. Therefore, it is quite possible that the acute induction of $c$-fos is mediated primarily through transient histone modifications, such as phosphorylation, whereas its chronic desensitization is more likely to be driven by more stable histone modifications, such as histone methylation. 
Although ample data have suggested an important role for transient histone modifications in the regulation of drug-induced behaviors, only recently has the considerably more stable modification, histone methylation, been demonstrated as an essential mediator of druginduced transcriptional, behavioral and synaptic plasticity ${ }^{59}$. As previously mentioned, genome-wide ChIP-chip analyses revealed that histone acetylation patterns were altered by chronic cocaine administration and that the specific histone methylation marks, histone 3 lysines 9 and 27 di-methylation (H3K9me2 and $\mathrm{H} 3 \mathrm{~K} 27 \mathrm{me} 2$ ), displayed regulation at numerous gene promoters by the drug ${ }^{40}$.

To further investigate these findings, a transcriptional profiling approach was taken to ask if excpression of specific histone methylating or demethylating enzymes was altered following repeated cocaine treatment. Of all of the histone "writers/erasers" examined, only two enzymes, G9a (Ehmt2) and GLP (Ehmt1), displayed persistent regulation following both repeated experimenter-delivered and self-administered cocaine exposure. Both HMTs were significantly downregulated in the NAc, indicating a potential role for these enzymes in the development of cocaine-induced behaviors ${ }^{59}$. Both G9a and GLP are ubiquitously expressed HMTs that specifically catalyze the mono- and di-methylated state of H3K9 (H3K9me1 and $\mathrm{H} 3 \mathrm{~K} 9 \mathrm{me} 2)^{60,61}$. These marks are correlated with transcriptional repression and are found primarily throughout euchromatic regions of the genome. In vivo, G9a and GLP form heteromeric binding complexes and display overlapping functions ${ }^{61}$. Recently, both of these enzymes have been linked to neural activity. They act to restrict neuronal gene expression to neurons and, when disrupted throughout the limbic forebrain, promote cognitive deficits reminiscent of a mental retardation syndrome in humans ${ }^{62-64}$. Consistent with G9a and GLP downregulation following chronic cocaine treatment, $\mathrm{H} 3 \mathrm{~K} 9 \mathrm{me} 1$ and $\mathrm{H} 3 \mathrm{~K} 9 \mathrm{me} 2$ were also globally reduced in the NAc with no alterations in global levels of total histone $\mathrm{H}^{59}$.

As mentioned earlier, although many cocaine-induced alterations in gene expression appear transiently after drug exposure, numerous genes display enhanced transcriptional inducibility (i.e., sensitization) following repeated drug administration. This phenomenon can be largely dependent on G9a activity in the NAc (Figure 4A) ${ }^{59}$. These data suggest that as G9a is repressed following chronic, but not acute, cocaine administration, G9a target genes become more sensitive (i.e., more likely to be transcriptionally induced) by future exposures to the drug. Furthermore, NAc-specific knockout of G9a, which mimics the effects of chronic cocaine exposure, enhances drug-associated reward behavior (Figure 3C). On the other hand, viral-mediated overexpression of G9a blunts this response, an event that has been shown to be dependent on G9a's catalytic activity ${ }^{59}$.

To identify potential mechanisms through which G9a is repressed by chronic cocaine exposure, as well as to identify putative G9a target genes regulated in response to cocaine treatment, a negative feedback loop was identified. In response to acute cocaine exposure, G9a acts to maintain homeostatic levels of gene expression in the NAc by repressing genes known to enhance synaptic plasticity in this brain region. However, following chronic treatment with the drug, G9a recruitment to target gene promoters is diminished, leading to the enhanced transcriptional inducibility/expression of associated transcripts ${ }^{59}$. This process was demonstrated to be dependent on $\triangle$ FosB accumulation following chronic cocaine treatment. Acute exposure to cocaine dramatically increased G9a binding to the fosb promoter, thus rapidly repressing cocaine-induced increases in $\triangle \mathrm{FosB}$ expression. Following repeated cocaine exposure, however, $\triangle \mathrm{FosB}$ accumulation in the NAc results in G9a repression and reduced global levels of $\mathrm{H} 3 \mathrm{~K} 9 \mathrm{me} 2$, preventing G9a's ability to maintain normal levels of gene expression and enhancing behavioral responses to the drug (see Figure 4B for schematic) ${ }^{59}$. Such inability to repress synaptic plasticity-related gene expression following chronic cocaine administration can result in enhanced dendritic spine plasticity in the NAc, an event which correlates with increased behavioral sensitivity to the drug and can 
be rescued by G9a overexpression in this brain region (Figure $4 \mathrm{C}$ ) ${ }^{65}$. Interestingly, $\Delta \mathrm{FosB}$ overexpression in the NAc, in the absence of cocaine, is sufficient to reduce G9a recruitment to target gene promoters and promotes increased dendritic spine density in this brain region. These phenomena can be potentiated by subsequent exposure to cocaine. These findings marked some of the first evidence of a role for chromatin regulation in the structural/ morphological changes observed following repeated cocaine exposure, likely contributing to enhancements in drug-induced behavioral sensitivity.

Surprisingly, given the stability of the modification and the likelihood of its involvement in mediating drug-induced transcriptional responses, very little is known concerning the role of DNA methylation in the development of persistent addictive-like behaviors. Recent studies examining global patterns of DNA methylation in the hippocampus of neonatal and prepubertal animals following in utero cocaine exposure indicate that altered levels of DNA methylation in this brain region may be important in regulating persistent patterns of druginduced gene expression, which may ultimately contribute to enhanced behavioral sensitivity later in life ${ }^{66}$. In accordance with these findings, the methyl-binding protein, $\mathrm{MeCP} 2$, has also been shown to be induced in cortical regions, as well as in the striatum, following chronic cocaine self-administration, correlating with changes in gene expression observed post-drug treatment ${ }^{49}$. More recently, a direct role for the DNA methylating enzyme, DNA methyltransferase 3a (DNMT3a) has been demonstrated. Reduced DNMT3a activity in the NAc, either by pharmacological inhibition or via viral-mediated knockdown of the enzyme, results in increased behavioral responses to cocaine (Figure 3D) ${ }^{33}$. Since DNMT3a generally functions to repress gene transcription, these data further support the hypothesis that aberrant increases in gene expression following repeated cocaine administration promote increased behavioral sensitivity to the drug. Interestingly, however, DNMT3a, unlike the HMT G9a or the histone deacetylases HDAC5 and HDAC2, promotes increased dendritic spine density in the NAc following cocaine administration $33,59,67$. Such paradoxical findings likely reflect "homeostatic adaptations," which compensate for additional alterations caused by repeated cocaine exposure, such as a reduction in glutamatergic stimulation of NAc neurons by prefrontal cortical afferents ${ }^{68,69}$. On the other hand, given that DNMT3a overexpression alone was sufficient to enhance NAc spine density, in combination with observations that these changes were not potentiated further by cocaine exposure, it is possible that such overexpression results in a form of metaplasticity, in which increased levels of DNMT3a expression in this brain region promote elevated structural plasticity, thus superseding additional alterations in spine density resulting from chronic cocaine administration. These data, as well as other examples of proteins known to increase NAc dendritic spine density but reduce behavioral responses to cocaine (e.g., Cdk5 and MEF2), highlight the complexity of these intracellular pathways and the importance of future research ${ }^{70-72}$. It will be important to further examine the involvement of such enzymes in controlling global genomic DNA methylation patterns in response to drugs of abuse, as well as to further investigate the potential roles of gene-specific DNA methylation profiles in the development of drug-induced behavioral and synaptic plasticity.

\section{Future research and conclusions}

Remarkable progress has been made in documenting distinct regulatory patterns of chromatin modifications and the subsequent activity of transcription factors and their downstream targets in response to administration of several drugs of abuse. However, many important questions still remain regarding the impact and persistence of such events in the development and maintenance of the drug addicted state in both animal models of abuse and in human addicts as well as possible effects of different classes of abused substances on chromatin endpoints. 
Although epigenetic mechanisms represent attractive candidates to explain long-lasting, and potentially even permanent, alterations in neuronal function following chronic drug exposure, it is still unclear as to how long after drug exposure these changes in chromatin structure persist. As mentioned previously, many of the genes known to display altered expression levels following repeated drug administration do not remain elevated or repressed during periods of extended withdrawal. Thus it appears necessary to shift the focus of current research to more thoroughly examine alterations in the transcriptional inducibility of gene targets following drug/cue re-exposure, as well as the epigenetic phenomena underlying such events. Doing so may aid in the identification of novel gene targets, which could prove useful for the development of future drug therapies. Furthermore, given that drug addiction is a highly heritable illness, it will be interesting to further examine whether drug-induced alterations in gene expression at the level of chromatin are likewise transmittable to future generations. This would involve stable, drug-induced epigenetic modifications in germ cells, which persist in offspring and affect addiction vulnerability. Whether such modifications occur remains a subject of intense investigation.

Another important question will be to ask on which genes, and in which regions of the genome, are drug-induced changes in chromatin structure occurring, as well as how these chromatin remodeling events ultimately affect the activity of genes in response to drug exposure? Although attempts have been made to address these questions through the use of genome-wide promoter analyses (ChIP-chip) and microarray technologies, the advent of next generation massively parallel genomic sequencing techniques (e.g., ChIP-Seq, RNASeq, etc.) promise enhanced DNA/RNA sequence-level resolution of chromatin binding events and transcriptional expression patterns $\mathrm{s}^{40,73,74}$. When examined at the level of numerous histone modifications (i.e., comparing combinatorial patterns of various histone marks), these techniques will allow for a stronger characterization of the genomic landscape following drug exposure. They also will permit future investigations into both the effects of drug treatment on gene expression, as well as the impact of chromatin regulation at noncoding genomic sequences (i.e., miRNAs, retrotransposons, enhancer elements, etc.), thus teasing apart another layer of complexity in our understanding of potential molecular mechanisms guiding addictive-like behavioral responses.

High-throughput genomic analyses of chromatin regulation, as well as targeted approaches, such as those described throughout this review, will further address questions concerning the importance of many other types of chromatin modifications (e.g., sumoylation, nucleosome remodeling and ubiquitination, to name a few) to drug addiction, and will aid in future investigations examining the similarities and/or differences between chromatin-mediated gene regulation in response to various drugs of abuse throughout numerous brain regions implicated in addiction. Such studies will allow for a fuller understanding of the ways in which drug-induced gene expression profiles throughout numerous limbic forebrain regions affect neural connectivity and electrophysiological communication between associated structures.

Lastly, it is important to consider that many of the brain regions affected by drug of abuse do not exist as homogeneous cell populations, but rather as heterogeneous structures composed of many distinct cell types with different signaling outputs and electrophysiological properties. For example, although the NAc is composed of approximately $95 \%$ medium spiny, GABAergic projection neurons receiving common inputs from several afferent pathways (e.g., glutamatergic, dopaminergic, etc.), these neurons express distinct dopamine receptor subtypes-dopamine receptor 1 (Drd1) and 2 (Drd2), which promote opposing downstream signaling cascades leading to very different transcriptional outcomes and, oftentimes, quite distinct behavioral responses to drugs of abuse $^{75-79}$. Analogous studies are needed for the several types of interneurons present in the 
NAc as well as glial cells. Therefore, it will be necessary to further examine drug-induced chromatin modifications in a cell type specific manner to more fully understand the contribution of differential modes of transcriptional plasticity in distinct cell types that may be involved in the development of addictive behaviors.

In conclusion, although our understanding of the molecular mechanisms underlying drug addiction remains incomplete, the identification of chromatin remodeling as an important mediator of drug-induced transcriptional and behavioral plasticity represents an exciting new area of research with potential therapeutic benefits. The ability to reverse the epigenetic landscape controlling the addicted state offers an approach that may aid in the development of more effective treatments for addiction, not only through direct targeting of aberrant chromatin regulation, but also through the future identification of target genes involved in addiction pathogenesis.

\section{References}

1. Hyman SE, Malenka RC, Nestler EJ. Neural mechanisms of addiction: the role of reward-related learning and memory. Annu Rev Neurosci. 2006; 29:565-598. [PubMed: 16776597]

2. Kalivas PW, Volkow N, Seamans J. Unmanageable motivation in addiction: a pathology in prefrontal-accumbens glutamate transmission. Neuron. 2005; 45:647-650. [PubMed: 15748840]

3. Koob G, Kreek MJ. Stress, dysregulation of drug reward pathways, and the transition to drug dependence. Am J Psychiatry. 2007; 164:1149-1159. [PubMed: 17671276]

4. Bird A. Perceptions of epigenetics. Nature. 2007; 447:396-398. [PubMed: 17522671]

5. Waddington, $\mathrm{CH}$., editor. The Strategy of the Genes; a Discussion of Some Aspects of Theoretical Bilogy. Allen, \& Unwin; London: 1957.

6. Holliday R, Pugh JE. DNA modification mechanisms and gene activity during development. Science. 1975; 187:226-232. [PubMed: 1111098]

7. Chambon P. Summary: the molecular biology of the eukaryotic genome is coming of age. Cold Spring Harb Symp Quant Biol. 1978; 42(Pt 2):1209-1234. [PubMed: 209932]

8. Jaenisch R, Bird A. Epigenetic regulation of gene expression: how the genome integrates intrinsic and environmental signals. Nat Genet. 2003; 33(Suppl):245-254. [PubMed: 12610534]

9. Surani MA, Hayashi K, Hajkova P. Genetic and epigenetic regulators of pluripotency. Cell. 2007; 128:747-762. [PubMed: 17320511]

10. Borrelli E, Nestler EJ, Allis CD, Sassone-Corsi P. Decoding the epigenetic language of neuronal plasticity. Neuron. 2008; 60:961-974. [PubMed: 19109904]

11. Berger SL. The complex language of chromatin regulation during transcription. Nature. 2007; 447:407-412. [PubMed: 17522673]

12. Cheung P, Allis CD, Sassone-Corsi P. Signaling to chromatin through histone modifications. Cell. 2000; 103:263-271. [PubMed: 11057899]

13. Strahl BD, Allis CD. The language of covalent histone modifications. Nature. 2000; 403:41-45. [PubMed: 10638745]

14. Luger K, Mader AW, Richmond RK, Sargent DF, Richmond TJ. Crystal structure of the nucleosome core particle at 2.8 A resolution. Nature. 1997; 389:251-260. [PubMed: 9305837]

15. Jenuwein T, Allis CD. Translating the histone code. Science. 2001; 293:1074-1080. [PubMed: 11498575]

16. Taverna SD, Li H, Ruthenburg AJ, Allis CD, Patel DJ. How chromatin-binding modules interpret histone modifications: lessons from professional pocket pickers. Nat Struct Mol Biol. 2007; 14:1025-1040. [PubMed: 17984965]

17. Goldberg AD, Allis CD, Bernstein E. Epigenetics: a landscape takes shape. Cell. 2007; 128:635638. [PubMed: 17320500]

18. Ruthenburg AJ, Li H, Patel DJ, Allis CD. Multivalent engagement of chromatin modifications by linked binding modules. Nat Rev Mol Cell Biol. 2007; 8:983-994. [PubMed: 18037899] 
19. Jaenisch R. DNA methylation and imprinting: why bother? Trends Genet. 1997; 13:323-329. [PubMed: 9260519]

20. Kouzarides T. Chromatin modifications and their function. Cell. 2007; 128:693-705. [PubMed: 17320507]

21. Pokholok DK, et al. Genome-wide map of nucleosome acetylation and methylation in yeast. Cell. 2005; 122:517-527. [PubMed: 16122420]

22. Brami-Cherrier K, Lavaur J, Pages C, Arthur JS, Caboche J. Glutamate induces histone H3 phosphorylation but not acetylation in striatal neurons: role of mitogen- and stress-activated kinase-1. J Neurochem. 2007; 101:697-708. [PubMed: 17241117]

23. Li J, et al. Dopamine D2-like antagonists induce chromatin remodeling in striatal neurons through cyclic AMP-protein kinase A and NMDA receptor signaling. J Neurochem. 2004; 90:1117-1131. [PubMed: 15312167]

24. Rice JC, Allis CD. Histone methylation versus histone acetylation: new insights into epigenetic regulation. Curr Opin Cell Biol. 2001; 13:263-273. [PubMed: 11343896]

25. Pinskaya M, Morillon A. Histone H3 lysine 4 di-methylation: a novel mark for transcriptional fidelity? Epigenetics. 2009; 4:302-306. [PubMed: 19633430]

26. Bernstein E, et al. Mouse polycomb proteins bind differentially to methylated histone H3 and RNA and are enriched in facultative heterochromatin. Mol Cell Biol. 2006; 26:2560-2569. [PubMed: 16537902]

27. Suzuki MM, Bird A. DNA methylation landscapes: provocative insights from epigenomics. Nat Rev Genet. 2008; 9:465-476. [PubMed: 18463664]

28. Zhang X, et al. Genome-wide analysis of cAMP-response element binding protein occupancy, phosphorylation, and target gene activation in human tissues. Proc Natl Acad Sci U S A. 2005; 102:4459-4464. [PubMed: 15753290]

29. Amir RE, et al. Rett syndrome is caused by mutations in X-linked MECP2, encoding methyl-CpGbinding protein, 2. Nat Genet. 1999; 23:185-188. [PubMed: 10508514]

30. Skene PJ, et al. Neuronal MeCP2 is expressed at near histone-octamer levels and globally alters the chromatin state. Mol Cell. 2010; 37:457-468. [PubMed: 20188665]

31. Chahrour M, et al. MeCP2, a key contributor to neurological disease, activates and represses transcription. Science. 2008; 320:1224-1229. [PubMed: 18511691]

32. Renthal W, Nestler EJ. Epigenetic mechanisms in drug addiction. Trends Mol Med. 2008; 14:341350. [PubMed: 18635399]

33. LaPlant, Q.e.a. Dnmt3a regulates emotional behavior and spine plasticity in the nucleus accumbens. 2010 In Press.

34. Kumar A, et al. Chromatin remodeling is a key mechanism underlying cocaine-induced plasticity in striatum. Neuron. 2005; 48:303-314. [PubMed: 16242410]

35. Brami-Cherrier K, et al. Parsing molecular and behavioral effects of cocaine in mitogen- and stress-activated protein kinase-1-deficient mice. J Neurosci. 2005; 25:11444-11454. [PubMed: 16339038]

36. Black YD, et al. Altered attention and prefrontal cortex gene expression in rats after binge-like exposure to cocaine during adolescence. J Neurosci. 2006; 26:9656-9665. [PubMed: 16988036]

37. Bibb JA, et al. Effects of chronic exposure to cocaine are regulated by the neuronal protein Cdk5. Nature. 2001; 410:376-380. [PubMed: 11268215]

38. Grimm JW, et al. Time-dependent increases in brain-derived neurotrophic factor protein levels within the mesolimbic dopamine system after withdrawal from cocaine: implications for incubation of cocaine craving. J Neurosci. 2003; 23:742-747. [PubMed: 12574402]

39. Freeman WM, et al. Persistent alterations in mesolimbic gene expression with abstinence from cocaine self-administration. Neuropsychopharmacology. 2008; 33:1807-1817. [PubMed: 17851536]

40. Renthal W, et al. Genome-wide analysis of chromatin regulation by cocaine reveals a role for sirtuins. Neuron. 2009; 62:335-348. [PubMed: 19447090] 
41. Tsankova NM, Kumar A, Nestler EJ. Histone modifications at gene promoter regions in rat hippocampus after acute and chronic electroconvulsive seizures. J Neurosci. 2004; 24:5603-5610. [PubMed: 15201333]

42. Renthal W, et al. Histone deacetylase 5 epigenetically controls behavioral adaptations to chronic emotional stimuli. Neuron. 2007; 56:517-529. [PubMed: 17988634]

43. Schroeder FA, et al. Drug-induced activation of dopamine $\mathrm{D}(1)$ receptor signaling and inhibition of class I/II histone deacetylase induce chromatin remodeling in reward circuitry and modulate cocaine-related behaviors. Neuropsychopharmacology. 2008; 33:2981-2992. [PubMed: 18288092]

44. Kalda A, Heidmets LT, Shen HY, Zharkovsky A, Chen JF. Histone deacetylase inhibitors modulates the induction and expression of amphetamine-induced behavioral sensitization partially through an associated learning of the environment in mice. Behav Brain Res. 2007; 181:76-84. [PubMed: 17477979]

45. Sanchis-Segura C, Lopez-Atalaya JP, Barco A. Selective boosting of transcriptional and behavioral responses to drugs of abuse by histone deacetylase inhibition. Neuropsychopharmacology. 2009; 34:2642-2654. [PubMed: 19727068]

46. Malvaez M, Sanchis-Segura C, Vo D, Lattal KM, Wood MA. Modulation of chromatin modification facilitates extinction of cocaine-induced conditioned place preference. Biol Psychiatry. 2010; 67:36-43. [PubMed: 19765687]

47. McQuown SC, Wood MA. Epigenetic regulation in substance use disorders. Curr Psychiatry Rep. 2010; 12:145-153. [PubMed: 20425300]

48. Levine AA, et al. CREB-binding protein controls response to cocaine by acetylating histones at the fosB promoter in the mouse striatum. Proc Natl Acad Sci U S A. 2005; 102:19186-19191. [PubMed: 16380431]

49. Host L, Dietrich JB, Carouge D, Aunis D, Zwiller J. Cocaine self-administration alters the expression of chromatin-remodelling proteins; modulation by histone deacetylase inhibition. $\mathrm{J}$ Psychopharmacol. 2009

50. Romieu P, et al. Histone deacetylase inhibitors decrease cocaine but not sucrose self-administration in rats. J Neurosci. 2008; 28:9342-9348. [PubMed: 18799668]

51. Sun J, et al. The effects of sodium butyrate, an inhibitor of histone deacetylase, on the cocaine- and sucrose-maintained self-administration in rats. Neurosci Lett. 2008; 441:72-76. [PubMed: 18599214]

52. Wang L, et al. Chronic cocaine-induced $\mathrm{H} 3$ acetylation and transcriptional activation of CaMKIIalpha in the nucleus accumbens is critical for motivation for drug reinforcement. Neuropsychopharmacology. 2010; 35:913-928. [PubMed: 20010550]

53. Russo SJ, et al. Nuclear factor kappa B signaling regulates neuronal morphology and cocaine reward. J Neurosci. 2009; 29:3529-3537. [PubMed: 19295158]

54. Finkel T, Deng CX, Mostoslavsky R. Recent progress in the biology and physiology of sirtuins. Nature. 2009; 460:587-591. [PubMed: 19641587]

55. Lu L, Koya E, Zhai H, Hope BT, Shaham Y. Role of ERK in cocaine addiction. Trends Neurosci. 2006; 29:695-703. [PubMed: 17084911]

56. Stipanovich A, et al. A phosphatase cascade by which rewarding stimuli control nucleosomal response. Nature. 2008; 453:879-884. [PubMed: 18496528]

57. Nestler EJ. Review. Transcriptional mechanisms of addiction: role of DeltaFosB. Philos Trans R Soc Lond B Biol Sci. 2008; 363:3245-3255. [PubMed: 18640924]

58. Renthal W, et al. Delta FosB mediates epigenetic desensitization of the c-fos gene after chronic amphetamine exposure. J Neurosci. 2008; 28:7344-7349. [PubMed: 18632938]

59. Maze I, et al. Essential role of the histone methyltransferase G9a in cocaine-induced plasticity. Science. 2010; 327:213-216. [PubMed: 20056891]

60. Tachibana M, Sugimoto K, Fukushima T, Shinkai Y. Set domain-containing protein, G9a, is a novel lysine-preferring mammalian histone methyltransferase with hyperactivity and specific selectivity to lysines 9 and 27 of histone H3. J Biol Chem. 2001; 276:25309-25317. [PubMed: 11316813] 
61. Tachibana M, et al. Histone methyltransferases G9a and GLP form heteromeric complexes and are both crucial for methylation of euchromatin at H3-K9. Genes Dev. 2005; 19:815-826. [PubMed: 15774718]

62. Ding N, et al. Mediator links epigenetic silencing of neuronal gene expression with x-linked mental retardation. Mol Cell. 2008; 31:347-359. [PubMed: 18691967]

63. Schaefer A, et al. Control of cognition and adaptive behavior by the GLP/G9a epigenetic suppressor complex. Neuron. 2009; 64:678-691. [PubMed: 20005824]

64. Balemans MC, et al. Reduced exploration, increased anxiety, and altered social behavior: Autisticlike features of euchromatin histone methyltransferase 1 heterozygous knockout mice. Behav Brain Res. 2010; 208:47-55. [PubMed: 19896504]

65. Robinson TE, Kolb B. Persistent structural modifications in nucleus accumbens and prefrontal cortex neurons produced by previous experience with amphetamine. J Neurosci. 1997; 17:84918497. [PubMed: 9334421]

66. Novikova SI, et al. Maternal cocaine administration in mice alters DNA methylation and gene expression in hippocampal neurons of neonatal and prepubertal offspring. PLoS One. 2008; 3:e1919. [PubMed: 18382688]

67. Guan JS, et al. HDAC2 negatively regulates memory formation and synaptic plasticity. Nature. 2009; 459:55-60. [PubMed: 19424149]

68. Berglind WJ, Whitfield TW Jr. LaLumiere RT, Kalivas PW, McGinty JF. A single intra-PFC infusion of BDNF prevents cocaine-induced alterations in extracellular glutamate within the nucleus accumbens. J Neurosci. 2009; 29:3715-3719. [PubMed: 19321768]

69. Thomas MJ, Beurrier C, Bonci A, Malenka RC. Long-term depression in the nucleus accumbens: a neural correlate of behavioral sensitization to cocaine. Nat Neurosci. 2001; 4:1217-1223. [PubMed: 11694884]

70. Pulipparacharuvil S, et al. Cocaine regulates MEF2 to control synaptic and behavioral plasticity. Neuron. 2008; 59:621-633. [PubMed: 18760698]

71. Norrholm SD, et al. Cocaine-induced proliferation of dendritic spines in nucleus accumbens is dependent on the activity of cyclin-dependent kinase-5. Neuroscience. 2003; 116:19-22. [PubMed: 12535933]

72. Benavides DR, et al. Cdk5 modulates cocaine reward, motivation, and striatal neuron excitability. J Neurosci. 2007; 27:12967-12976. [PubMed: 18032670]

73. Park PJ. ChIP-seq: advantages and challenges of a maturing technology. Nat Rev Genet. 2009; 10:669-680. [PubMed: 19736561]

74. Barski A, Zhao K. Genomic location analysis by ChIP-Seq. J Cell Biochem. 2009; 107:11-18. [PubMed: 19173299]

75. Surmeier DJ, Ding J, Day M, Wang Z, Shen W. D1 and D2 dopamine-receptor modulation of striatal glutamatergic signaling in striatal medium spiny neurons. Trends Neurosci. 2007; 30:228235. [PubMed: 17408758]

76. Lobo MK. Molecular profiling of striatonigral and striatopallidal medium spiny neurons past, present, and future. Int Rev Neurobiol. 2009; 89:1-35. [PubMed: 19900613]

77. Chen BT, Hopf FW, Bonci A. Synaptic plasticity in the mesolimbic system: therapeutic implications for substance abuse. Ann N Y Acad Sci. 2010; 1187:129-139. [PubMed: 20201850]

78. Heiman M, et al. A translational profiling approach for the molecular characterization of CNS cell types. Cell. 2008; 135:738-748. [PubMed: 19013281]

79. Self DW, Barnhart WJ, Lehman DA, Nestler EJ. Opposite modulation of cocaine-seeking behavior by D1- and D2-like dopamine receptor agonists. Science. 1996; 271:1586-1589. [PubMed: 8599115] 


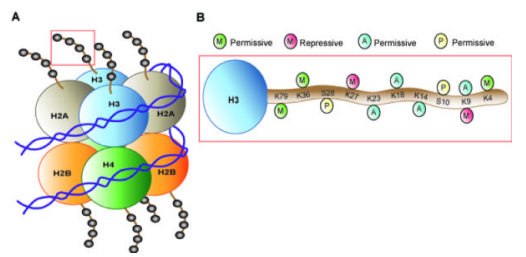

Figure 1.

Post-translational modifications of histones regulate gene expression. Shown in panel (A) is the nucleosome core particle, representing the functional repeating unit of chromatin, composed of $147 \mathrm{bp}$ of DNA wrapped around a core octamer of histone proteins (two copies each of H2A, H2B, H3 and H4). (B) Combinations of acetylation, phosphorylation, methylation, etc. on histone tails (here, $\mathrm{H} 3$ is depicted) alter chromatin compaction promoting altered levels of gene expression in cells. Histone modifications that weaken the interaction between histones and DNA (e.g., histone acetylation at K23, K18, K14, and K9, as well as methylation at $\mathrm{K} 79, \mathrm{~K} 36$, and $\mathrm{K} 4$ or phosphorylation at S28 and S10) correlate with permissive gene expression. Histone deacetylation or histone methylation on H3K27 or H3K9, which strengthen histone:DNA contacts, promote a state of transcriptional repression. Figure 1 (adapted) from Maze I. \& Russo, S.J. 2010. Transcriptional mechanisms underlying addiction-related structural plasticity. Molecular Interventions. In Press. with permission. 


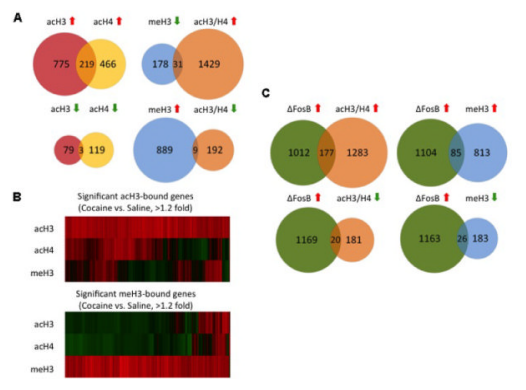

Figure 2.

Chronic cocaine exposure alters genome-wide patterns of histone modifications and transcription factor binding in NAc. (A) Venn diagrams and (B) heat maps depicting genes displaying altered levels of $\mathrm{H} 3$ or $\mathrm{H} 4$ acetylation and $\mathrm{H} 3$ methylation (dimethyl-K9/K27) promoter occupancy $24 \mathrm{hr}$ after chronic cocaine administration. (C) Venn diagrams of genes displaying significant promoter binding of the transcription factor $\Delta \mathrm{FosB}$, and/or of $\mathrm{H} 3 / \mathrm{H} 4$ acetylation or $\mathrm{H} 3$ methylation in NAc, following chronic cocaine administration. Figure 2 (adapted) from ${ }^{40}$ with permission. 

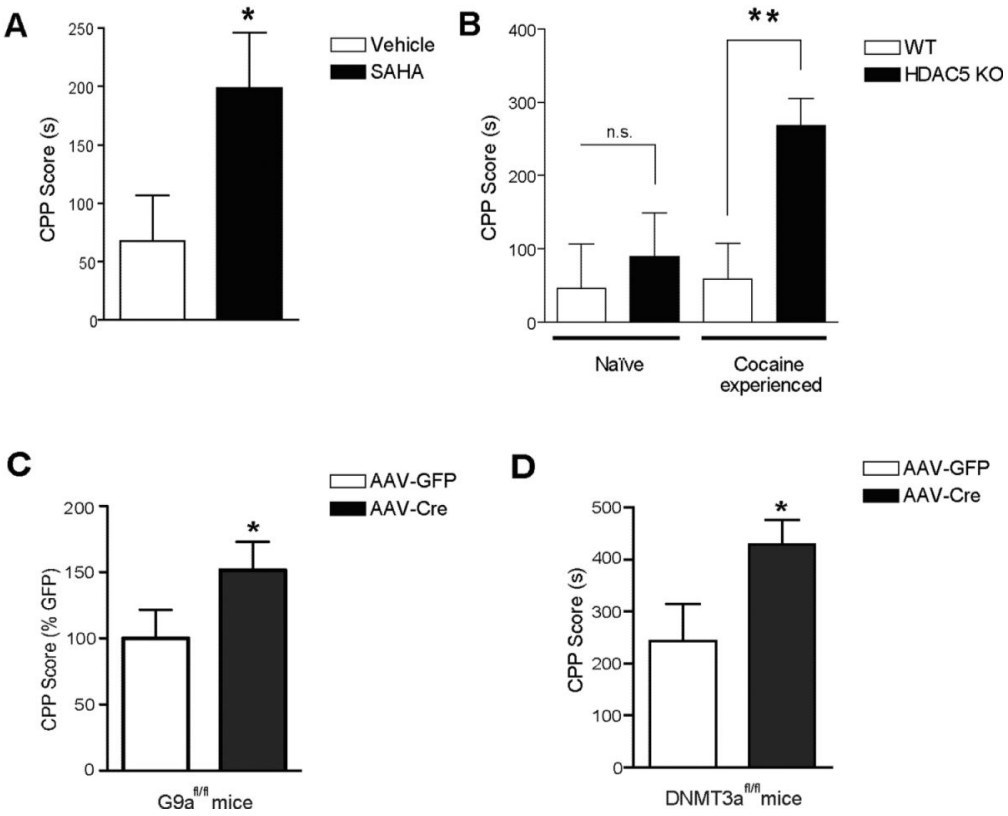

Figure 3.

Cocaine-induced disruption of repressive histone modifying enzymes in NAc results in enhanced behavioral plasticity. (A) Intra-NAc delivery of the specific HDACi suberoylanilide hydroxamic acid (SAHA) significantly potentiates conditioned place preference (CPP) for cocaine. (B) Cocaine-naive HDAC5 KO mice do not show a greater preference for cocaine in comparison to their wildtype littermate controls, however, after prior exposure to cocaine, HDAC5 KO mice develop a significant hypersensitization to cocaine reward. Viral-mediated knockdown of (C) the histone methyltransferase G9a or (D) the DNA methyltransferase DNMT3a in NAc, results in significantly enhanced CPP for cocaine. Figure 3 (adapted) from ${ }^{33(D), 42(A \& B), 59(C)}$ with permission. 


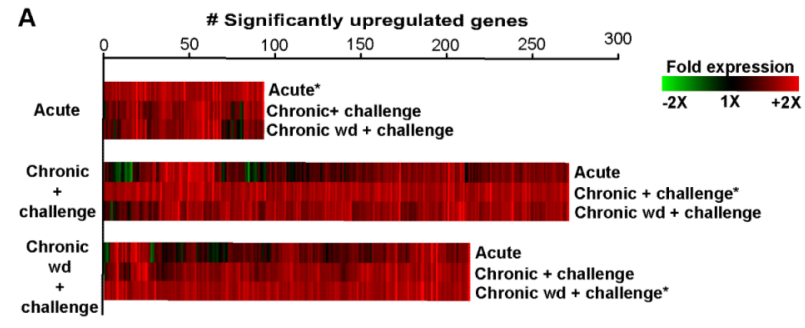

B

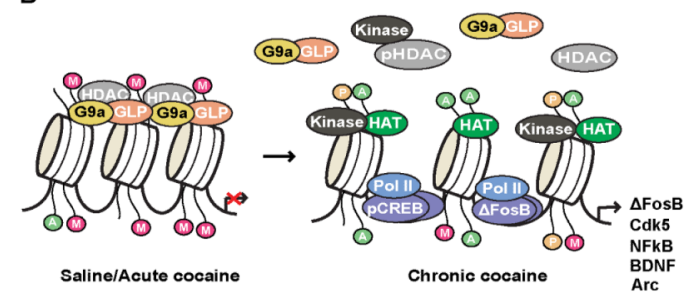

C 口Saline

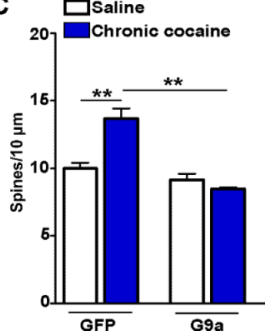

Figure 4.

Chronic cocaine administration increases both transcriptional and structural plasticity in NAc through chromatin dependent mechanisms. (A) Analysis of gene expression after acute or repeated cocaine indicates increased transcriptional activation/inducibility in NAc. Heat maps (*) display genes up-regulated in NAc 1 hour after a cocaine challenge in naïve animals (acute), in animals treated repeatedly with cocaine (chronic + challenge), or in animals after 7 days of withdrawal from chronic cocaine (chronic wd + challenge). Associated heat maps show how genes are affected under the other two conditions. (B) Methylation of H3K9 promotes chromatin condensation and gene repression. Following either saline or acute cocaine exposure, a complex of HMTs, mainly G9a and GLP, along with HDACs (e.g., HDAC5) bind to histones and repress transcription. After chronic cocaine administration, however, repressive HMT and HDAC binding to plasticity related gene promoters is reduced, which, in combination with increased kinase activity and binding of HATs, $\triangle$ FosB, pCREB and RNA polymerase II (Pol II), results in a permissive state of transcription. Such decreased binding of repressive chromatin complexes results in increased structural plasticity on NAc MSNs. (C) Overexpression of G9a in NAc, which opposes the endogenous effects of cocaine, blocks cocaine-induced alterations in transcription and significantly reduces structural plasticity in this brain region. Figure 4 (adapted) from 59(A \& C) and Maze I. \& Russo, S.J. 2010. Transcriptional mechanisms underlying addiction-related structural plasticity. Molecular Interventions. In Press. with permission. 